\title{
Phototactic Algae-Driven Unidirectional Transport of Submillimeter-Sized Cargo in a Microchannel
}

\author{
Moeto Nagai *(D), Takahiro Hirano and Takayuki Shibata \\ Department of Mechanical Engineering, Toyohashi University of Technology, Toyohashi, Aichi 441-8580, Japan; \\ saucy_cat1030@yahoo.co.jp (T.H.); shibata@me.tut.ac.jp (T.S.) \\ * Correspondence: nagai@me.tut.ac.jp; Tel.: +81-532-44-6701
}

Received: 18 January 2019; Accepted: 8 February 2019; Published: 16 February 2019

\begin{abstract}
The sensing and actuation capabilities of biological cells integrated with artificial components have been used to create autonomous microsystems. For creating autonomous microsystems, the unidirectional transport of a submillimeter-sized cargo with stimuli responsive bio-motors should be developed as a fundamental motion. This study aims to use Volvox as a light-controlled microrobot to achieve the unidirectional transport of a submillimeter-sized cargo. We show the fabrication of a guide structure, cargo, and light irradiation platform for a unidirectional actuation. The fundamental performances of each component were investigated, and the motions of Volvox were controlled in a microchamber with the developed light irradiation platform. All components were integrated to demonstrate the unidirectional actuation of a block by Volvox. We discuss the dynamics of the mechanical motions.
\end{abstract}

Keywords: microrobot; phototaxis; Volvox; micro-swimmer; biohybrid system; motion control

\section{Introduction}

Controllable micro-robots are essential for tasks in the micro scale such as transportation, regulation, and assembly. Such micro-robots are still difficult to fabricate artificially using current microfabrication technologies, because these technologies rely on two-dimensional processes [1,2]. Artificial micro-/nanomotors have been produced from asymmetric spherical and tubular structures and recent studies demonstrated light-controlled movements [3-5] and taxis-behaviors [6,7]; nevertheless, they often require the precise regulation of an operating environment. Natural micro-robots in living organisms can stably work under a wider range of environmental conditions and are more sophisticated than artificial ones. These biological micro-robots perform multiple functions of motor, sensory receptor, and metabolism [8]. To take full advantage of their sensing and actuation capabilities, biological cells have been integrated with artificial components to create bio-hybrid systems. These investigations have led to the development of novel microsystems and the increased functionality of them. Cell actuators have powered autonomous bio-hybrid tweezers [9], mixers [10,11], gear [12], and pumps [13,14] in the microscale.

Biological motors are responsive to external signals, and the motions of living organisms have been controlled using magnetic [15-19], electrical [20-23], chemical [24-27], and optical [12,23,28-34] methods. Among them, the optical methods are advantageous in that cellular motions are controlled remotely without contacting the cells, which provides light-controlled or light-responsive systems. Light-sensitive motors are expected to be a micro-control unit with an embedded light sensor for autonomous microsystems.

Regardless of the motion control of biological motors, micrometer to millimeter objects have been transported by unicellular and multicellular micro-swimmers. Micrometer objects were transported by unicellular organisms such as Escherichia coli ( 2 $\mu \mathrm{m}$ in length) [27,35-37], Magnetococcus marinus 
(1-2 $\mu \mathrm{m}$ in diameter) [18], Serratia marcescens (several micrometers in length) [23,30], bovine sperm (10 $\mu \mathrm{m}$ in length) [38], and Chlamydomonas reinhardtii (10 $\mu \mathrm{m}$ in diameter) [31]. To actuate an order of magnitude for a larger object or more than the size of unicellular micro-swimmers, their collective motion should be used [23,30,37]. Multicellular micro-swimmers spontaneously express the collective motion of somatic cells, and they are more convenient for actuating submillimeter to millimeter objects than unicellular micro-swimmers. Millimeter objects were driven by multicellular organism such as Volvox aureus (200-500 $\mu \mathrm{m}$ in diameter) [32] and Artemia salina ( several mm in length) [33,34]. Specifically, a gear was floated without a shaft, and it was rotated and translated by V. aureus [32] and A. salina [33]. A. salina moved a millimeter float along a guide [34]. The unidirectional transport of a submillimeter-sized cargo without rotation is a fundamental mechanism and in demand for applications in autonomous microsystems, yet it has not been achieved.

Among micro-swimmers, Volvox carteri is suitable for actuating sub-mm objects, because Volvox shows positive phototaxis and swims by means of flagella on thousands of surface somatic cells [39]. Moreover, V. carteri has a diameter of approximately $200 \mu \mathrm{m}$ [40] and its total swimming force is $\sim 1 \mathrm{nN}$ [41]. Since a pusher-type swimmer creates a flow field away from the cell [42], nearby pushers can transport cargo. On the other hand, the velocity field around $V$. carteri shows it is a neutral swimmer $[43,44]$. We hypothesize that $V$. carteri should be adjacent to the wall of a block to actuate a cargo in the swimming direction. The objective of this study is to use Volvox as a light-controlled microrobot and achieve the unidirectional transport of a submillimeter-sized cargo. This achievement requires the combination use of Volvox, a guide structure, cargo, and a light irradiation platform. In this paper, we prepare a guide structure, cargo, and light irradiation platform for a unidirectional actuation by Volvox. We investigate the fundamental performance of each component. All components are integrated to demonstrate the unidirectional actuation by Volvox and the mechanical motions are characterized.

\section{Materials and Methods}

\subsection{Overview of Experimental Setup}

Figure 1 shows the experimental setup used in this study. We developed a common light irradiation platform and set two types of micro chambers where $V$. carteri and a microblock were introduced. This platform allowed light irradiation from four directions by using LEDs of a, b, c, and d. We selected a green LED (emission maximum centered at $\lambda=525 \mathrm{~nm}$, outer diameter: $3 \mathrm{~mm}$, Stanley Electric, UG3803X, Tokyo, Japan) for controlling the motion of $V$. carteri. LED light was illuminated from one side to induce the migration of $V$. carteri.

The colonies have positive phototaxis and move toward light sources [45]. V. carteri (NIES-865) was obtained from the Microbial Culture Collection at the National Institute for Environmental Studies (NIES), Japan and cultured in either a MG medium (NIES) or algae universal medium (Wamushiya). $V$. carteri was illuminated by a white LED light in a daily cycle of $14 \mathrm{~h}$ of light and $10 \mathrm{~h}$ of darkness. The intensity of the growth light was 4000 lux.

A microchip was mounted on the stage of a stereo microscope (Olympus, SZ61, Tokyo, Japan). $V$. carteri was placed into the chamber and observed under the stereo microscope equipped with a camera (Point Grey Research, Grasshopper Express, Richmond, BC, Canada). The illumination light of the microscope was filtered with a red transmission filter to prevent the photo responses to the bright-field illumination from the halogen lamp. The motion of the colonies was analyzed using image analysis software (ImageJ, Version 1.52). 

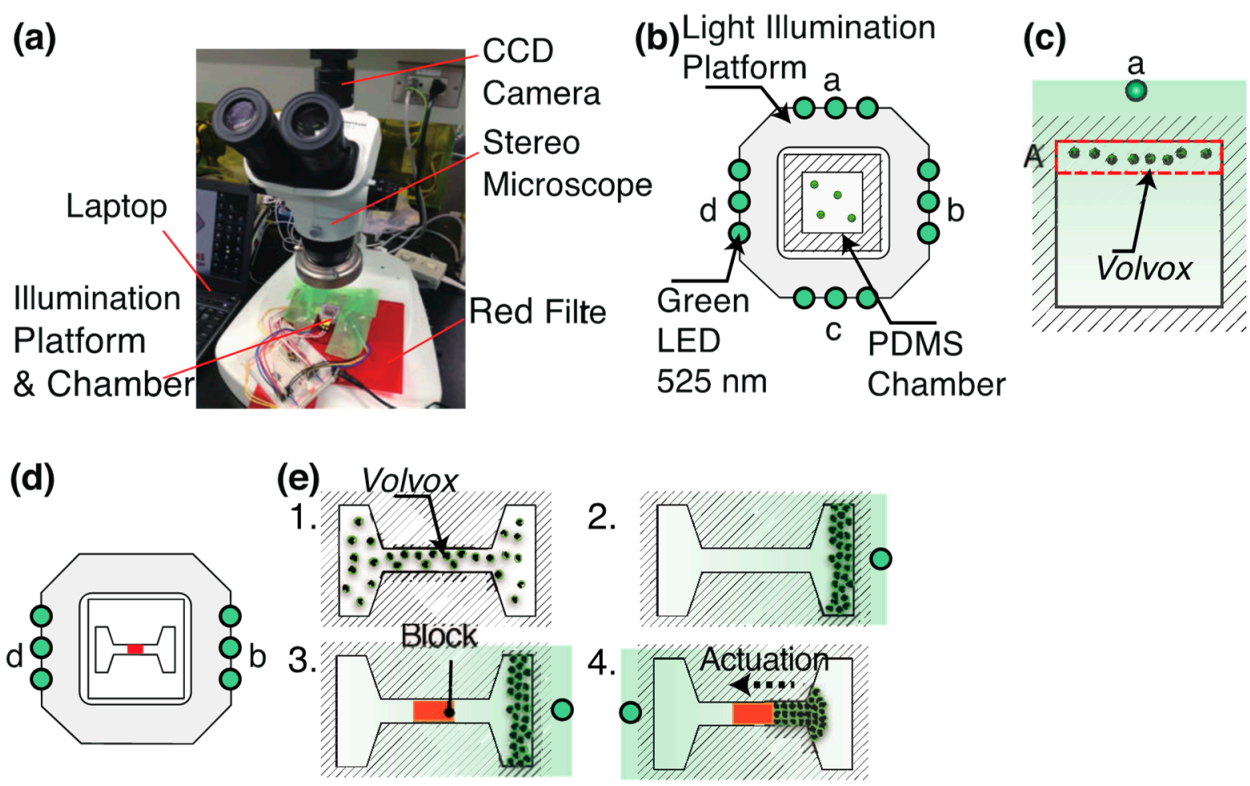

Figure 1. Experimental methods for the demonstration of (b), (c) phototactic control and (d), (e) unidirectional transport. (a) Picture of an experimental setup. (b), (d) schematic of the experimental setup. (c) Phototactic migration of Volvox to region A with LED $a$. (e) Experimental processes for the actuation of a movable block by $V$. carteri.

\subsection{Phototaxis and Swimming Speed Evaluation of Volvox carteri in Square Microchamber}

We controlled the motions of $V$. carteri by switching the LEDs approximately every $70 \mathrm{~s}$ (Figure $1 \mathrm{~b}, \mathrm{c})$. The chamber was divided into four regions, A, B, C, and D. The number of colonies in the regions was counted every $5 \mathrm{~s}$ to evaluate their phototaxis. We also measured each swimming distance, $\Delta d$ in time, $\Delta t . \Delta d / \Delta t$ gives the swimming speeds of Volvox colonies. 100 Volvox colonies were randomly selected from a recorded video. We plotted a distribution diagram of the swimming speeds of $V$. carteri.

We designed a square microchamber (vertical $9.4 \mathrm{~mm} \times$ horizontal $9.4 \mathrm{~mm} \times$ height $0.65 \mathrm{~mm}$ ) in AutoCAD and fabricated it in polydimethylsiloxane (PDMS, Silpot 184, Dow Corning Toray, Tokyo, Japan) by softlithography. PDMS base and curing agents were mixed in a weight ratio of 10:1. The detailed fabrication process is described in the Supplementary Materials. Briefly, a PDMS chamber was replicated from a printing plate (relief depth $0.65 \mathrm{~mm}$, Fujifilm, Torelief DWF95DTN, Toray Industries, Tokyo, Japan) and bonded to a glass substrate to form a closed PDMS chamber.

\subsection{Unidirectional Transport of the Block by Volvox}

Figure 1d,e show the experimental setup and procedures for transporting a block. Colonies of $V$. carteri and a movable block were placed in a PDMS chamber. The motions of Volvox colonies were controlled by switching between LED $b$ and LED $d$ on both sides of the platform to control their movements. Their driving forces transported the block unidirectionally.

We prepared this setup via the following steps: (1) An open top PDMS and block were fabricated according to the methods described in Section 2.4. They were immersed in a solution of surfactants (Pluronic ${ }^{\circledR}$ F-127, Sigma-Aldrich, St. Louis, MO, USA) to prevent the suction of the block to the PDMS channel. (2) The suspension of $V$. carteri in a culture medium was transferred to the chamber with a syringe. (3) The motion of the Volvox colonies was controlled by turning on LED $b$ or $d$ to migrate them to one side of the chamber. (4) While V. carteri colonies were trapped, a block was placed manually in the center of the channel with tweezers. (5) Volvox colonies were steered to the other side with LED $b$ or $d$ to actuate the block. (6) The block continued to experience the collective swimming motion of Volvox colonies and its motion was observed with a stereo microscope. The position of the block structure 
was recorded every $10 \mathrm{~s}$. The average driving speed of the block was measured from an actuation distance and time.

\subsection{Fabrication of Thick SU-8 Structure with a Rubber Ring}

We fabricated a submillimeter-sized thick SU-8 structure with a silicone rubber ring, which had a function of storing a thick SU-8 resist. Our method was based on SU-8 casting [46]. A rubber sheet with a thickness of $0.4 \mathrm{~mm}$ was patterned with a cutting machine (silhouette CAMEO, GRAPHTEC Corp., Yokohama, Japan) to fit a 3-inch diameter with a $2 \mathrm{~mm}$ width (Figure 2a). The rubber sheet was pasted in a wafer (Figure $2 b$ ).

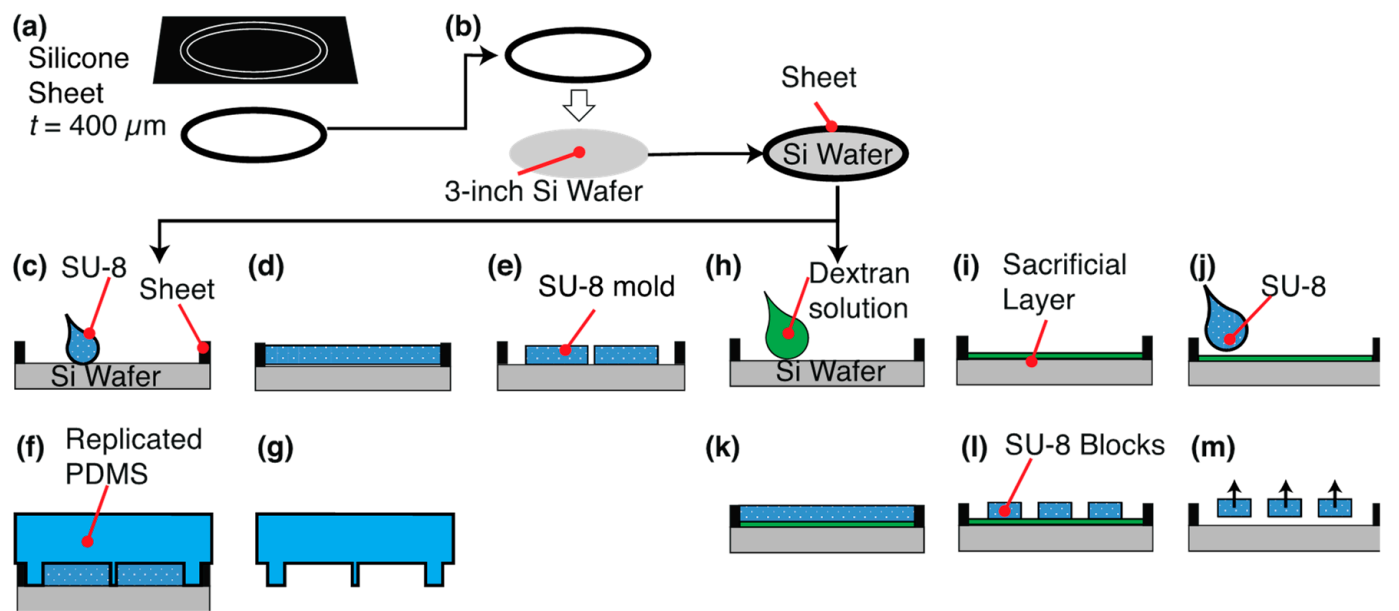

Figure 2. Schematic of a SU-8 mold fabrication process. (a) Ring formation from a silicone sheet. (b) Placement of a rubber sheet on a wafer. (c-g) Fabrication of a polydimethylsiloxane (PDMS) microchamber from a SU-8 mold. (c) SU-8 dispensing, (d) flattening by gravity. (e) SU-8 photolithography. (f) PDMS molding. (g) Release of the PDMS micro chamber. (h-m) Fabrication of the SU-8 blocks. (h) Dispensing of a dextran solution. (i) Spin-coating of the dextran. (j) SU-8 dispensing. (k) Flattening by gravity. (1) SU-8 photolithography. (m) Release of the SU-8 blocks in water.

We designed a photomask of a micro chamber and block, which were required for the unidirectional transportation of a block by V. carteri (Figure 3). One combination of a block and chamber is shown in Figure 3a. All patterns of the chambers and blocks are displayed in Figure 3b,c. We used SU-8 3050 (12,000 cSt, 75.5\% solids, Microchem, Newton, MA, USA) and calculated a required volume of SU-8. The original solution of SU-8 was diluted with cyclopentanone at a weight ratio of 1:1 to decrease its viscosity. This diluted SU-8 was poured onto a wafer with a ring and spread uniformly.

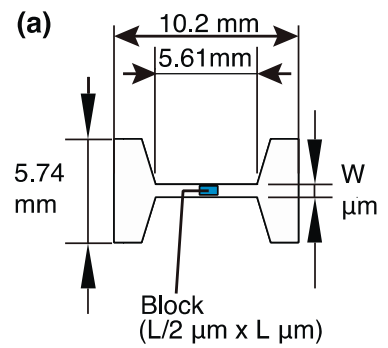

(b) (c)

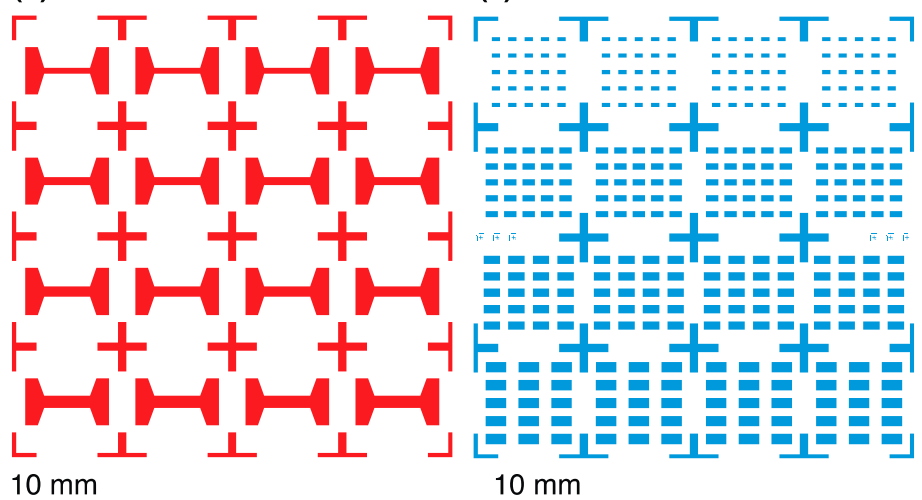

Figure 3. Design of a block and chamber for the actuation experiment by Volvox. (a) Dimensional drawing of a single block integrated in a chamber. Photomask patterns of (b) chambers and (c) blocks. 
The photolithography of a thick SU-8 resist was processed under the common following conditions. (1) SU-8 was prebaked on a hot plate to cure the coated resist at $65{ }^{\circ} \mathrm{C}(15 \mathrm{~min})$, $95^{\circ} \mathrm{C}(240 \mathrm{~min})$, and $65^{\circ} \mathrm{C}$ (15 min). (2) We used a double-sided aligner (PEM-800, Union Optical, Tokyo, Japan) and the wafer was exposed to reach a light integral of $2500 \mathrm{~mJ} / \mathrm{cm}^{2}$ through a polymethylmethacrylate (PMMA) filter (3) The resist was post-baked at $65^{\circ} \mathrm{C}(6 \mathrm{~min}), 95^{\circ} \mathrm{C}(15 \mathrm{~min})$, and $65^{\circ} \mathrm{C}(6 \mathrm{~min})$. (4) The SU-8 coated substrate was developed in 2-methoxy-1-methylethyl acetate for $15 \mathrm{~min}$. The SU-8 was rinsed in acetone and blown by a nitrogen gas.

\subsection{Fabrication of Open-Top PDMS Chamber and SU-8 Blocks}

A photomask of single micro chambers is shown in Figure 3b. One chamber is $5.5 \mathrm{~mm}$ in width and $10.2 \mathrm{~mm}$ in length. A flow channel with a length of about $5.6 \mathrm{~mm}$ was created for the rectified actuation of a block in the center. The sizes of the blocks were designed to be $500 \mu \mathrm{m} \times 1000 \mu \mathrm{m}$, $750 \mu \mathrm{m} \times 1500 \mu \mathrm{m}, 1000 \mu \mathrm{m} \times 2000 \mu \mathrm{m}$, and $1250 \mu \mathrm{m} \times 2500 \mu \mathrm{m}$ (Width $\times$ Length). To actuate these blocks, the gap between the block and a guide channel was set to $100 \mu \mathrm{m}$. Therefore, we prepared four types of channel widths, $0.7 \mathrm{~mm}, 0.95 \mathrm{~mm}, 1.2 \mathrm{~mm}$, and $1.45 \mathrm{~mm}$.

An open top PDMS chamber was fabricated using the following methods. (1) We weighed $2.5 \mathrm{~g}$ of SU-8 3050 to make a $300 \mu \mathrm{m}$ height mold and diluted SU-8 was poured on a silicon wafer (Figure 2c). (2) The resist was flattened by gravity (Figure 2d). (3) Photolithography (Figure 2e). (4) A $10 \mu \mathrm{L}$ of Trichloro $(1 \mathrm{H}, 1 \mathrm{H}, 2 \mathrm{H}, 2 \mathrm{H}$-perfluorooctyl)silane was vaporized and exposed to the SU-8 mold for ease of releasing. (5) PDMS (10:1 base:curing agent) mixture was poured over the SU-8 mold and air bubbles were removed in a vacuum chamber. (6) The PDMS was cured at $80^{\circ} \mathrm{C}$ for $2 \mathrm{~h}$ in an oven (Figure 2f). (7) The cured PDMS was peeled off from the mold (Figure 2g).

$250 \mu \mathrm{m}$ thick blocks were fabricated similarly to the microchamber by photolithography. The major difference is the spin-coating of a sacrificial layer on a silicon wafer before the resist coating. (1) $10 \%(\mathrm{w} / \mathrm{v})$ aqueous solution of dextran was spin-coated at $1000 \mathrm{rpm}$ for $20 \mathrm{~s}$ (Figure 2h). We selected a 10\% (w/v) dextran (Mw: 60,000) as a sacrificial layer. (2) A dextran film was formed on a hot plate at $150{ }^{\circ} \mathrm{C}$ for $2 \mathrm{~min}$ (Figure 2i). (3) We weighed $2.1 \mathrm{~g}$ of SU-8 3050 for the structure and diluted SU-8 was poured on the wafer (Figure 2j). (4) Photolithography (Figure 2k,1). (5) Release of the blocks in water (Figure $2 \mathrm{~m}$ ).

The dimensions of the structures were observed with a microscope (VHX-2000, KEYENCE, Osaka, Japan). The height of the chambers and blocks was measured by observing the cross-sections.

\section{Results and Discussion}

\subsection{Phototactic Steering of V. carteri in a Square Chamber}

Colonies of $V$. carteri were injected into a closed square chamber and migrated to a light irradiation direction. They followed the changes of the light directions switched approximately every $70 \mathrm{~s}$ (Figure 4). After reaching the edge near the lit LED, Volvox stayed in their places. Figure $4 \mathrm{a}-\mathrm{d}$ show migrated Volvox colonies in four regions, A, B, C, and D, where the time stamps in the figure correspond to the peak number in each condition. Figure 4e illustrates four conditions of Volvox in areas A-D with LEDs a-d. Figure 4f,g plot the number of Volvox colonies in the four regions taken at every five seconds. Light illumination increased the number of Volvox in the area closest to the lit LED and decreased the number in the opposite region. Approximately $50 \%$ of the total 214 colonies moved to the following region within about $70 \mathrm{~s}$. A typical distance from one region to the next region is $7 \mathrm{~mm}$. The speeds obtained from the time and travelling distance agreed with the swimming speeds of Volvox ranging $0 \mu \mathrm{m} / \mathrm{s}$ to about $350 \mu \mathrm{m} / \mathrm{s}$ [44]. The motion control experiments indicate the collective motion can be used for the transportation of a block. The number of Volvox colonies 214 and the volume of a chamber $57 \mathrm{~mm}^{3}(=9.4 \times \mathrm{mm} \times 9.4 \mathrm{~mm} \times 0.65 \mathrm{~mm})$ give the density of Volvox, 4 colonies $/ \mathrm{mm}^{3}$. 
We acquired swimming videos and evaluated the swimming speeds of 100 randomly selected Volvox colonies in a micro chamber. Figure 5 shows a histogram of a swimming speed distribution of $V$. carteri, which was calculated from each swimming distance and time. This distribution is bimodal and has two peaks at around $0 \mu \mathrm{m} / \mathrm{s}$ and $230 \mu \mathrm{m} / \mathrm{s}$. The average is approximately $170 \mu \mathrm{m} / \mathrm{s}$ and the mode is $230 \mu \mathrm{m} / \mathrm{s}$. The left peak can be explained by the rest of the horizontal movement of about $20 \%$ of the total Volvox, which remained at the same places. They were stuck or their motilities were naturally lower. This stoppage was most likely caused by the trapping of the Volvox between the PDMS and the glass substrates.

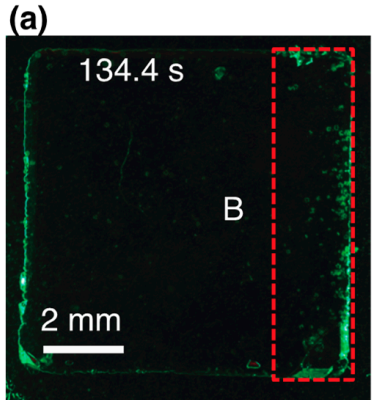

(c)

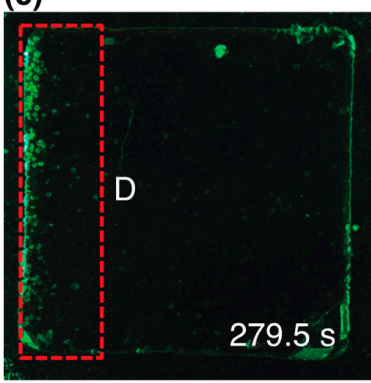

(e)

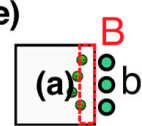

(b)

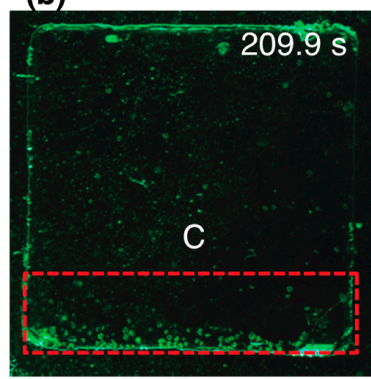

(d)
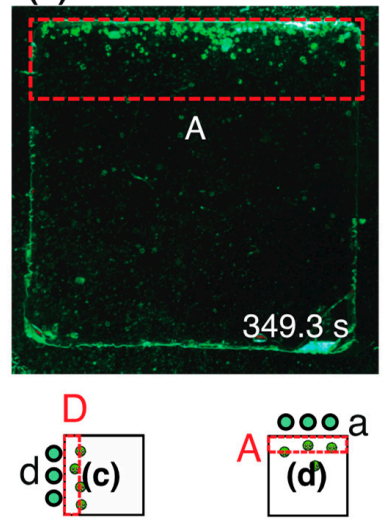

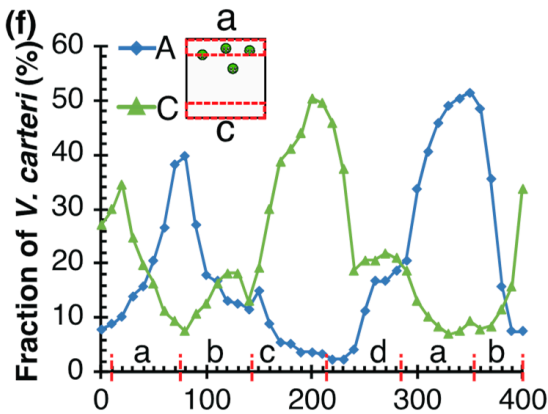

Time (s)

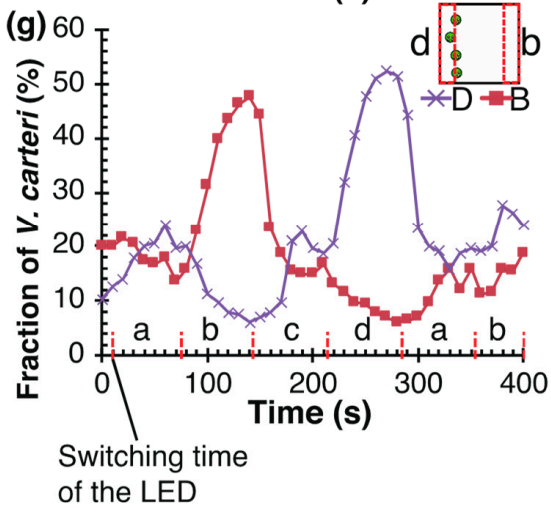

Figure 4. Motion control results of $V$. carteri in a closed micro chamber with the light irradiation platform. (a-d) Micrographs showing a state of motion control of $V$. carteri (green points). (e) Schematic of Volvox in areas A-D with LEDs a-d. (f), (g) time is plotted on the horizontal axis and the number of $V$. carteri is plotted on the vertical axis. These graphs separately plot A, C and B, D that face each other. Red dashed lines represent the switching time of the $\operatorname{LED}(a, b, c, d)$.

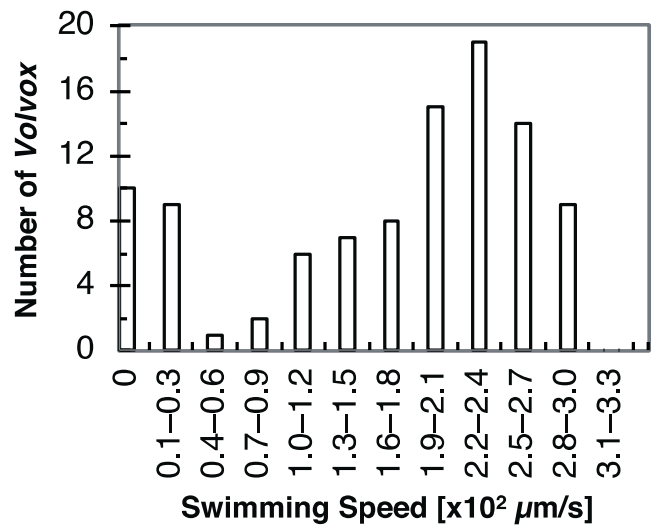

Figure 5. Swimming speed distribution of Volvox carteri calculated by the equation in Section 2.2. The horizontal axis is the swimming speeds and the vertical axis is the frequency of the population. 


\subsection{Fabrication of Thick Open-Top Chambers and SU-8 Blocks}

An open-top PDMS chamber and SU-8 blocks were fabricated with diluted SU-8 and a rubber ring. Thick SU-8 molds for a chamber were fabricated (Figure 6) and PDMS chambers were replicated from the molds. We measured the widths and thickness of the channels and plotted the width of channels in Figure 6e. The measurement results show that the mold was fabricated with a shrinkage of about $2-3 \%$ from the designed value. The PDMS chamber was cut with a razor and we measured the chamber height by observing the cross section with the microscope. We obtained a uniform height chamber with a height of $238.9 \pm 22.1 \mu \mathrm{m}$ (mean \pm standard deviation, $\mathrm{n}=35$ points). Dilution of the resist lowered the viscosity of SU-8 and enabled uniform thickness of the chamber.
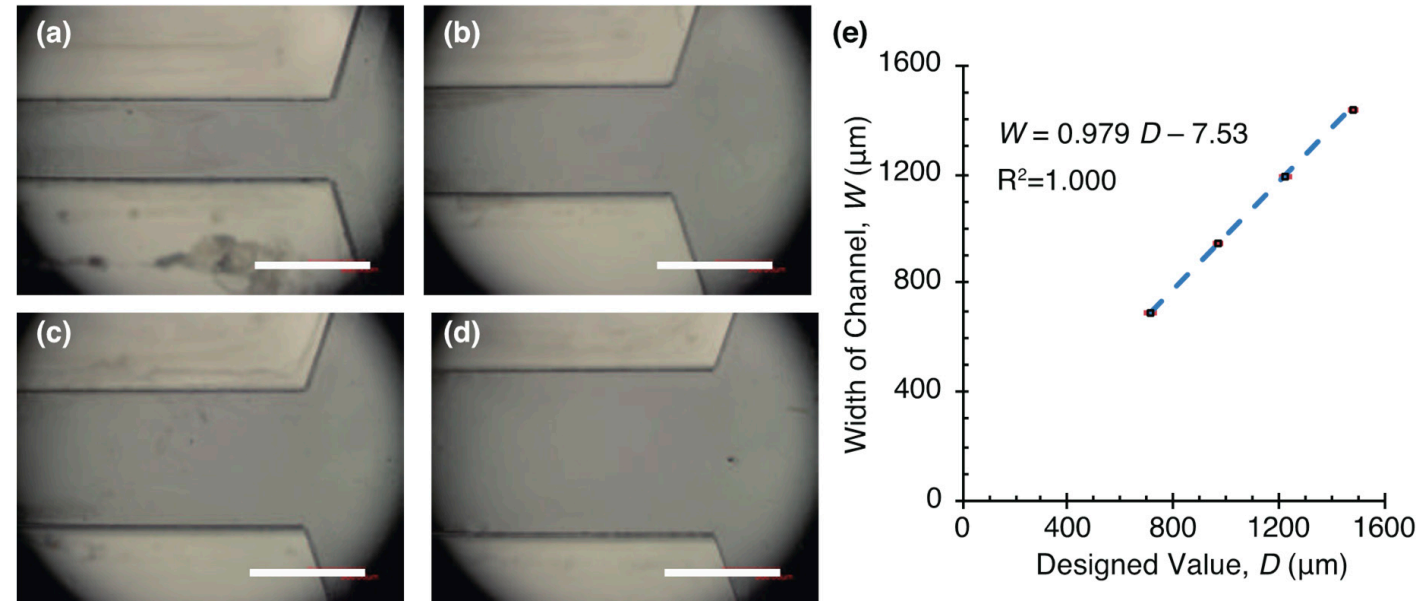

Figure 6. Fabrication of thick SU-8 molds for an open-top microchamber. Microscope image of channels with widths of (a) $714 \mu \mathrm{m}$, (b) $969 \mu \mathrm{m}$, (c) $1225 \mu \mathrm{m}$, and (d) $1480 \mu \mathrm{m}$. Scale bars: $1 \mathrm{~mm}$. (e) Actual widths of SU-8 channels versus designed values.

Four patterns of submillimeter-sized SU-8 blocks were fabricated (Figure 7) and released from the wafer after dissolution of a dextran layer. The thickness of the blocks was $282.9 \pm 23.9 \mu \mathrm{m}(\mathrm{N}=20)$. This block thickness fits the height of the PDMS channel. Figure 7a shows SU-8 blocks on a dextran layer and Figure $7 \mathrm{~b}$ gives the yield of released blocks. The major reason of lowering the yield is the insufficient formation of a sacrificial layer on a wafer. This yield can be improved with uniform coating of dextran by surface treatment before spin-coating. Figure $7 \mathrm{c}-\mathrm{f}$ present microscope images of each block. The measurement results of the block width and length are plotted in Figure 7g,h. The length and width of blocks were almost the same as the design values.

\subsection{Unidirectional Transport of a Block by V. carteri}

The suspension of $V$. carteri colonies were injected into an open top chamber. The LEDs on one side were used to control the motion of the colonies and migrated them to the side of the chamber. Figure 8 shows the placement of an SU-8 block in the center of the channel using tweezers, while the colonies migrated to the right. This manual handling did not break the SU-8 blocks. Since SU-8 has higher density [47] than water, the block sedimented in water and were in a stationary state. We confirmed a drift flow was negligible in an open top chamber. A chamber was in a steady state $3 \mathrm{~s}$ after a disturbance was manually exerted on the chamber. 

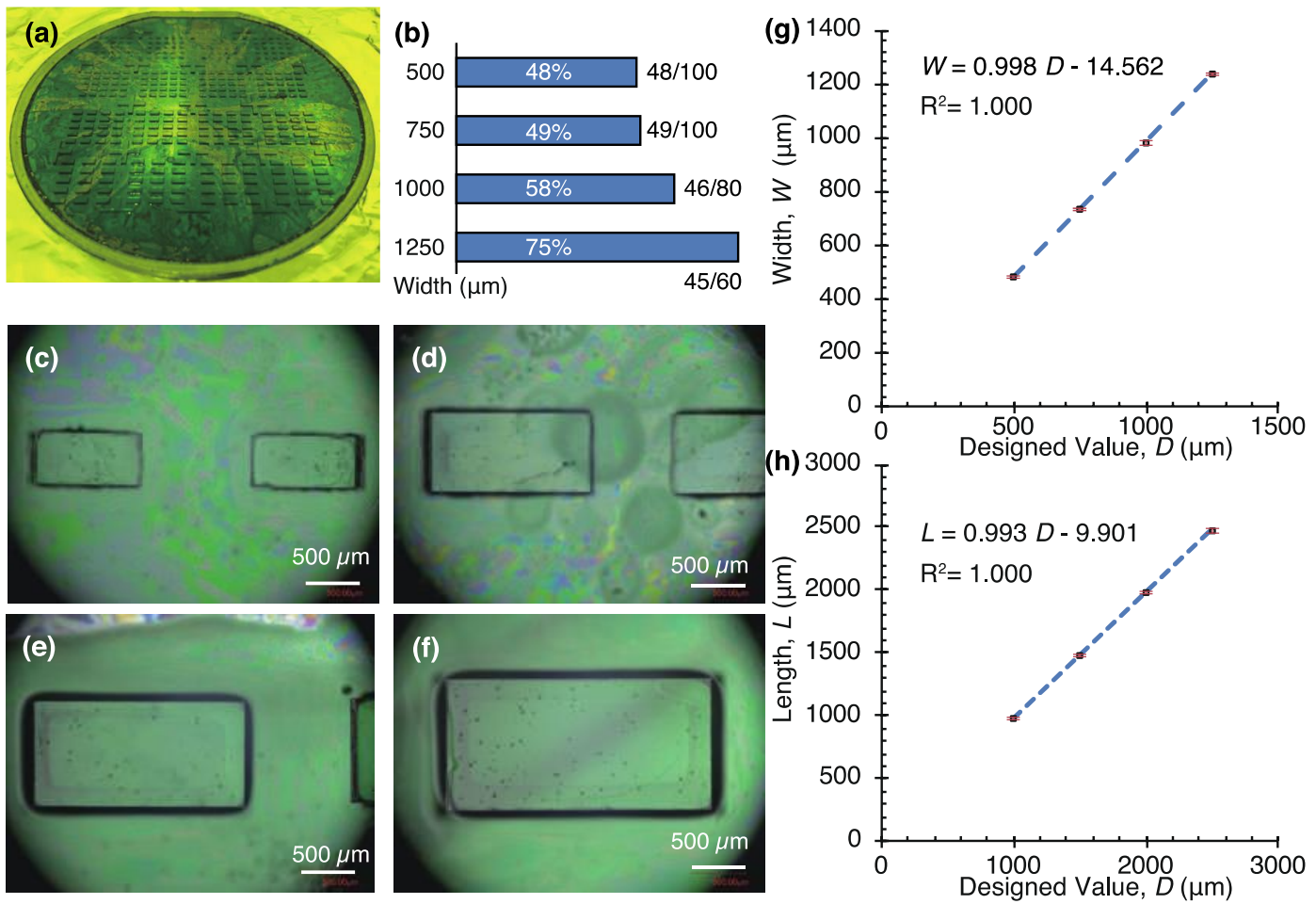

Figure 7. Fabrication results of the SU-8 blocks. (a) Entire view. (b) Yield of released blocks. Microscope images of blocks with designed values of (c) $500 \mu \mathrm{m} \times 1000 \mu \mathrm{m}$, (d) $750 \mu \mathrm{m} \times 1500 \mu \mathrm{m}$, (e) $1000 \mu \mathrm{m} \times 2000 \mu \mathrm{m}$, and (f) $1250 \mu \mathrm{m} \times 2500 \mu \mathrm{m}$ (Width $\times$ Length). (g) Widths and (h) lengths of SU-8 blocks versus designed values.
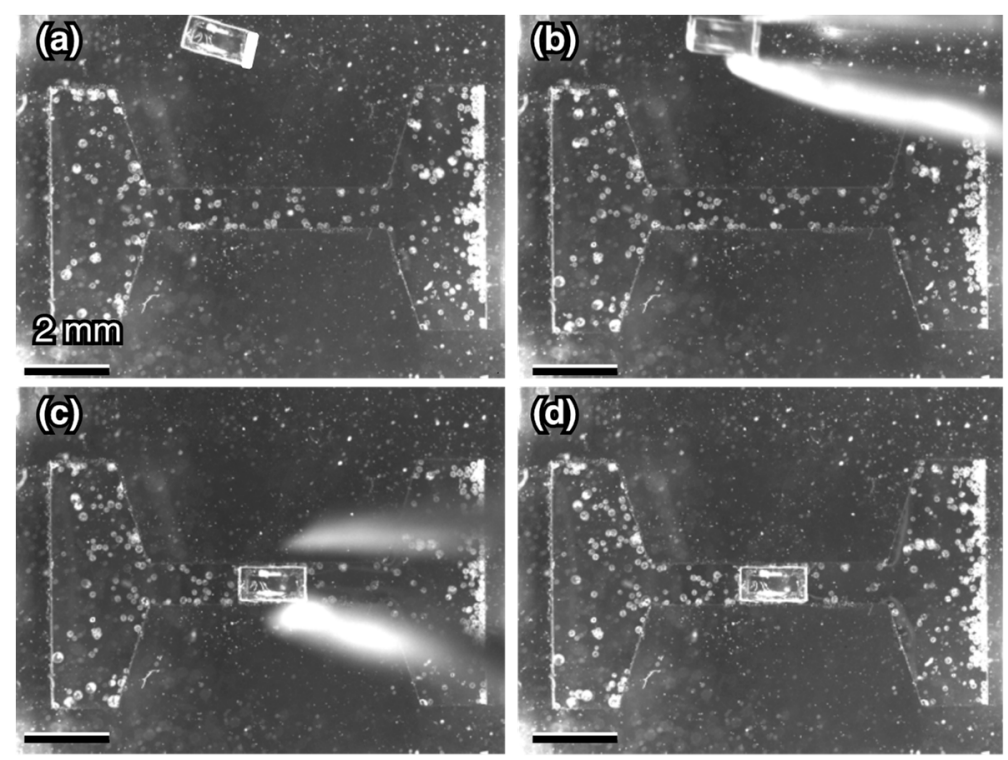

Figure 8. Placement of a $750 \mu \mathrm{m}$ width block in a $970 \mu \mathrm{m}$ width micro channel with tweezers. (a) Initial position of the block. (b) Pickup of the block. (c) Positioning of the block at the center of the channel. (d) Final position of the block.

We demonstrated the unidirectional transportation of a block in a guide channel using phototaxis in Volvox (Figure 9). 3 of the 5 trials with a $500-\mu \mathrm{m}$ width block and 1 of 5 trials with a $750 \mu \mathrm{m}$ width block were transported by $V$. carteri. Figure 9a,b and Supplementary Movie S1 show the migration of $V$. carteri to the left irradiation direction and the movement of a block toward the left side. About 10 colonies were adjacent on the right side of the $500 \mu \mathrm{m}$ width block, around 50 colonies 
were accumulated behind the adjacent colonies. Even when the almost same number of colonies collided with the block while pushing it, the block was intermittently actuated. About 10 colonies were sandwiched between the block and guide and they are presumed to be obstacles. Without the collision of Volvox, a block was not transported. Figure $9 \mathrm{c}$ is the travelling lengths of actuated blocks. The experiments were labelled in descending order of travelling lengths. The driving speeds were calculated from the structure driving distance in each of these sections, and the fastest driving speed was $24 \mu \mathrm{m} / \mathrm{s}$ at around $240 \mathrm{~s}$ in experiment \#1. The adhesion of the block to the channel was prevented by coating them with Pluronic. Compared with the rotation and translation of a gear by V. aureus [32], we achieved the unidirectional transport of a cargo without rotation. This restricted motion was enabled by the combination use of Volvox, a guide structure, cargo, and light irradiation platform.

As the large variations in the travel distances and the step-like pattern were displayed in Figure $9 c$, the possible reasons are discussed. We consider resistance and driving forces on a block and characterize the dynamics of the transportation. The drag forces on a block are the viscous forces against the block and the friction forces between the block and a guide or $V$. carteri. When a bubble smaller than a block remained between a block and guide, the bubble pinned the block. In such a case, a pinning force should be considered. The driving forces are thrust generated by $V$. carteri. A cargo started to be transported by the Volvox colonies, when the total driving forces of $V$. carteri exceeded the total resistances forces on the cargo. On the other hand, a block stopped when the total restriction forces were greater than the total propulsive forces.
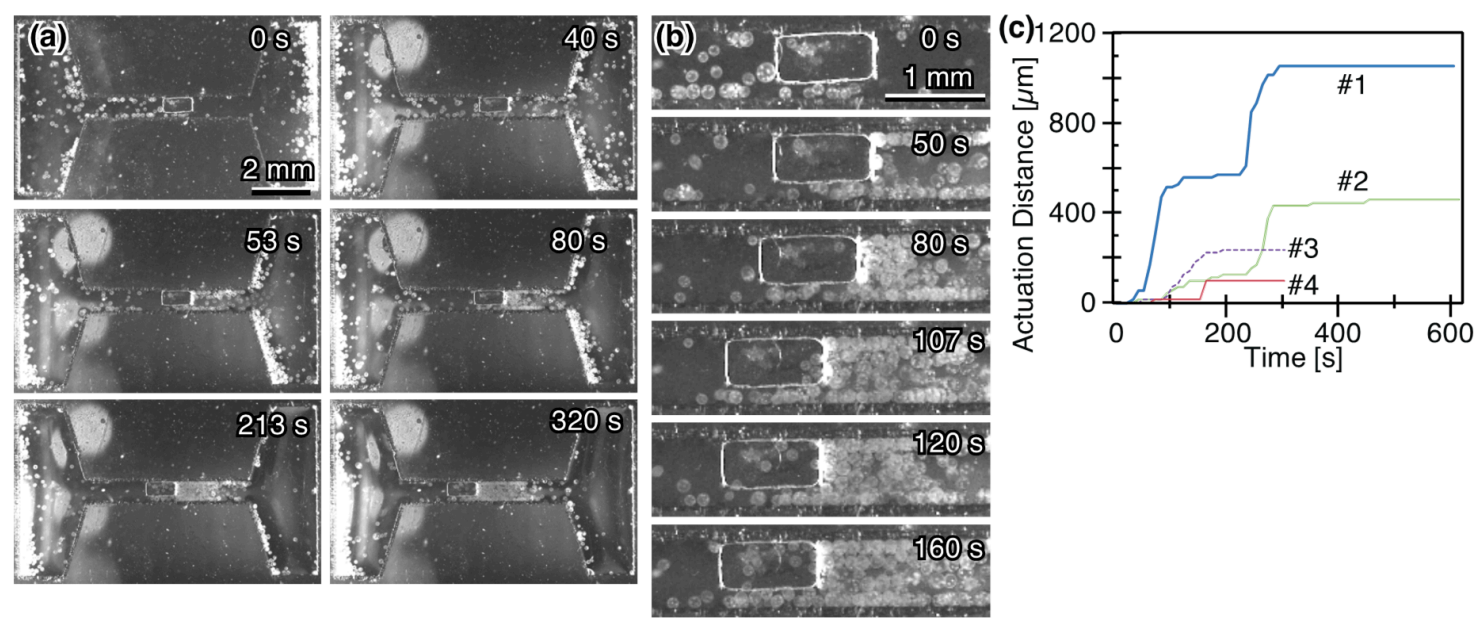

Figure 9. Unidirectional transport of a block in a microchannel. Time series micrographs showing the actuation results of a block in (a) the whole chamber and (b) the central region. A $500 \mu \mathrm{m}$ width block was put in a $710 \mu \mathrm{m}$ width channel. (c) Travel distances of the actuated blocks by $V$. carteri. The data were labeled as \#1-4 based on the travel length order. \#1-3 and \#4 were taken with a $500 \mu \mathrm{m}$ and $750 \mu \mathrm{m}$ width blocks in a $710 \mu \mathrm{m}$ and $970 \mu \mathrm{m}$ width channel, respectively.

$V$. carteri generates a horizontal driving force while $V$. carteri laterally swim. We calculate the viscous force on swimming Volvox in the fluid in the lateral direction. A swimming force of Volvox, F, is calculated considering Stokes' drag represented by the following formula.

$$
F=6 \pi \eta R V
$$

where $\eta$ is the viscosity of the medium, $R$ is the radius of $V$. carteri, and $V$ is swimming speed of $V$. carteri. The medium viscosity at $20^{\circ} \mathrm{C}$ is $\eta=1.0 \mathrm{mPa}$. The speed is obtained from the previous experiment in Section 3.1, and we assume $V=200 \mu \mathrm{m} / \mathrm{s}$. The average diameter of swimming Volvox was $2 R=140 \pm 20 \mu \mathrm{m}(\mathrm{N}=30)$. Substituting these values into Equation (1) gives the driving force of $V$. carteri, $F=0.26 \mathrm{nN}$. When 10 colonies push the block in one direction, the total force is simply calculated to be the sum of 10 colonies and in the order of $3 \mathrm{nN}$. This calculated force is probably 
overestimated because each $V$. carteri maintained a portion of its thrust when pushing the block and only a portion of the propulsive thrust of $V$. carteri was used to push the block. In practice, the swimming direction of all the colonies is not aligned for the entire time and this variation of the driving forces is considered to cause the intermittent actuation of a block.

\section{Conclusions}

We picked up a microalgae, Volvox carteri, and demonstrated the one-way transportation of a block based on its phototaxis, which allowed for the use of this algae as a microrobot. We evaluated the phototaxis and measured the swimming speeds of Volvox colonies in a closed square micro chamber. Approximately $50 \%$ of total colonies migrated to the area closest to the lit LED. We prepared a guided structure, cargo, and light irradiation platform for a unidirectional actuation. We established the fabrication process of a thick SU-8 mold and block with diluted SU-8 and the rubber sheet. A sacrificial layer was formed underneath the blocks, and the dextran layer was dissolved to release the blocks.

All components were integrated and used to achieve the block actuation. The block was intermittently transported by the swimming forces of the Volvox colonies. The dynamics of the block actuation was characterized and the restriction and driving forces on the block were calculated to understand the actuation principle. We observed the large variations in the travel distances and the step-like pattern, accordingly, the experimental setup should be improved to remove the effect of the restrictive forces. We hypothesized that $V$. carteri (neutral swimmer) should be adjacent to the wall of a block for actuating a cargo in the swimming direction. Adjacent $V$. carteri colonies transported the cargo unidirectionally by colliding with the cargo. We consider pusher swimmers transport a cargo at a distance from the cargo.

Our developed technologies are fundamental for accomplishing one-way actuation of a submillimeter-size cargo by Volvox. As our group reported a microfluidic valve based on the Volvox outer wall [48], we envisage higher-level applications in microfluidic systems with the use of positive phototaxis of microorganisms and an extension tool. Integration of this block actuation mechanism into the system will lead to broaden the application fields. Phototactic microrobots enable us to make smart microdevices that can change their functions dynamically by responding to an optical environment. According to the same principles of autonomous microsystems based on stimuli-responsive hydrogels [49], light-sensitive motors will bring autonomous microsystems such as fluidic valves, sorters, regulators, pumps, mixers, drug-delivery devices, fluidic cooling devices, and liquid lenses.

Supplementary Materials: The following are available online at http:/ / www.mdpi.com/2072-666X/10/2/130/ s1, Movie S1: The unidirectional actuation of a block in the whole chamber. This video is eight times faster than real time. The development of the light illumination platform and the fabrication method of a square micro-chamber are provided in the Supplementary data.

Author Contributions: M.N., T.H., and T.S. conceived and designed the experiments; M.N. and T.H. performed the experiments; M.N. and T.H. analyzed the experimental data; T.H. contributed reagents and materials; M.N. and T.H. wrote the paper.

Funding: This work was supported by the Hatakeyama Culture Foundation.

Conflicts of Interest: The authors declare no conflict of interest.

\section{References}

1. Franssila, S. Introduction to Microfabrication, 2nd ed.; John Wiley \& Sons: Chichester, UK, 2010; 518p.

2. Madou, M.J. Fundamentals of Microfabrication and Nanotechnology, 3rd ed.; CRC Press: Boca Raton, FL, USA, 2012.

3. Wang, J.; Xiong, Z.; Zheng, J.; Zhan, X.; Tang, J. Light-Driven Micro/Nanomotor for Promising Biomedical Tools: Principle, Challenge, and Prospect. Acc. Chem. Res. 2018, 51, 1957-1965.

4. Chen, C.; Mou, F.; Xu, L.; Wang, S.; Guan, J.; Feng, Z.; Wang, Q.; Kong, L.; Li, W.; Wang, J.; et al. Light-Steered Isotropic Semiconductor Micromotors. Adv. Mater. 2017, 29, 1603374. 
5. Xu, L.; Mou, F.; Gong, H.; Luo, M.; Guan, J. Light-driven micro/nanomotors: From fundamentals to applications. Chem. Soc. Rev. 2017, 46, 6905-6926.

6. You, M.; Chen, C.; Xu, L.; Mou, F.; Guan, J. Intelligent Micro/nanomotors with Taxis. Acc. Chem. Res. 2018, 51, 3006-3014.

7. Liebchen, B.; Löwen, H. Synthetic Chemotaxis and Collective Behavior in Active Matter. Acc. Chem. Res. 2018, 51, 2982-2990.

8. Alberts, B.; Wilson, J.H.; Hunt, T. Molecular Biology of the Cell, 5th ed.; Garland Science: New York, NY, USA, 2008; 1690p.

9. Akiyama, Y.; Sakuma, T.; Funakoshi, K.; Hoshino, T.; Iwabuchi, K.; Morishima, K. Atmospheric-operable bioactuator powered by insect muscle packaged with medium. Lab Chip 2013, 13, 4870-4880. [CrossRef] [PubMed]

10. Nagai, M.; Hayasaka, Y.; Kato, K.; Kawashima, T.; Shibata, T. Mixing of solutions by coordinated ciliary motion in Vorticella convallaria and patterning method for microfluidic applications. Sens. Actuators B 2013, 188, 1255-1262. [CrossRef]

11. Nagai, M.; Hayasaka, Y.; Kawashima, T.; Shibata, T. Active mixing in microchamber using cilia of Vorticella convallaria. IEEJ Trans. Electr. Electron. Eng. 2014, 9, 575-576. [CrossRef]

12. Vizsnyiczai, G.; Frangipane, G.; Maggi, C.; Saglimbeni, F.; Bianchi, S.; Di Leonardo, R. Light controlled 3d micromotors powered by bacteria. Nat. Commun. 2017, 8, 15974. [CrossRef] [PubMed]

13. Tanaka, Y.; Morishima, K.; Shimizu, T.; Kikuchi, A.; Yamato, M.; Okano, T.; Kitamori, T. An actuated pump on-chip powered by cultured cardiomyocytes. Lab Chip 2006, 6, 362-368. [CrossRef] [PubMed]

14. Kim, M.J.; Breuer, K.S. Microfluidic pump powered by self-organizing bacteria. Small 2008, 4, 111-118. [CrossRef] [PubMed]

15. Martel, S.; Tremblay, C.C.; Ngakeng, S.; Langlois, G. Controlled manipulation and actuation of micro-objects with magnetotactic bacteria. Appl. Phys. Lett. 2006, 89, 233904. [CrossRef]

16. Kim, D.H.; Cheang, U.K.; Kőhidai, L.; Byun, D.; Kim, M.J. Artificial magnetotactic motion control of tetrahymena pyriformis using ferromagnetic nanoparticles: A tool for fabrication of microbiorobots. Appl. Phys. Lett. 2010, 97, 173702. [CrossRef]

17. Ma, Q.; Chen, C.; Wei, S.; Chen, C.; Wu, L.-F.; Song, T. Construction and operation of a microrobot based on magnetotactic bacteria in a microfluidic chip. Biomicrofluidics 2012, 6, 024107. [CrossRef]

18. Felfoul, O.; Mohammadi, M.; Taherkhani, S.; de Lanauze, D.; Xu, Y.Z.; Loghin, D.; Essa, S.; Jancik, S.; Houle, D.; Lafleur, M.; et al. Magneto-aerotactic bacteria deliver drug-containing nanoliposomes to tumour hypoxic regions. Nat. Nanotechnol. 2016, 11, 941. [CrossRef]

19. Santomauro, G.; Singh, A.V.; Park, B.-W.; Mohammadrahimi, M.; Erkoc, P.; Goering, E.; Schütz, G.; Sitti, M.; Bill, J. Incorporation of terbium into a microalga leads to magnetotactic swimmers. Adv. Biosyst. 2018, 2, 1800039. [CrossRef]

20. Itoh, A. Motion control of protozoa for bio-mems. IEEE/ASME Trans. Mechatron. 2000, 5, 181-188. [CrossRef]

21. Kim, D.H.; Casale, D.; Kőhidai, L.; Kim, M.J. Galvanotactic and phototactic control of Tetrahymena pyriformis as a microfluidic workhorse. Appl. Phys. Lett. 2009, 94, 163901. [CrossRef]

22. Ogawa, N.; Oku, H.; Hashimoto, K.; Ishikawa, M. Microrobotic visual control of motile cells using high-speed tracking system. IEEE Trans. Robot. 2005, 21, 704-712. [CrossRef]

23. Edward, B.S.; Mahmut Selman, S.; Dal Hyung, K.; Vijay, K.; George, J.P.; Min Jun, K. Electrokinetic and optical control of bacterial microrobots. J. Micromech. Microeng. 2011, 21, 035001.

24. Nagai, M.; Ryu, S.; Thorsen, T.; Matsudaira, P.; Fujita, H. Chemical control of vorticella bioactuator using microfluidics. Lab Chip 2010, 10, 1574-1578. [CrossRef]

25. Nagai, M.; Matsumoto, N.; Kawashima, T.; Shibata, T. Reversible motion control of vorticella stalk in microchannel. Microelectron. Eng. 2013, 108, 28-32. [CrossRef]

26. Kim, M.J.; Breuer, K.S. Controlled mixing in microfluidic systems using bacterial chemotaxis. Anal. Chem. 2007, 79, 955-959. [CrossRef]

27. Park, B.-W.; Zhuang, J.; Yasa, O.; Sitti, M. Multifunctional bacteria-driven micro-swimmers for targeted active drug delivery. ACS Nano 2017, 11, 8910-8923. [CrossRef]

28. Kim, J.; Ennis, H.L.; Nguyen, T.H.; Zhuang, X.; Luo, J.; Yao, J.; Kessin, R.H.; Stojanovic, M.; Lin, Q. Light-directed migration of D. discoideum slugs in microfabricated confinements. Sens. Actuators A 2012, 188, 312-319. [CrossRef] 
29. Ozasa, K.; Lee, J.; Song, S.; Hara, M.; Maeda, M. Two-dimensional optical feedback control of euglena confined in closed-type microfluidic channels. Lab Chip 2011, 11, 1933-1940. [CrossRef] [PubMed]

30. Steager, E.; Kim, C.-B.; Patel, J.; Bith, S.; Naik, C.; Reber, L.; Kim, M.J. Control of microfabricated structures powered by flagellated bacteria using phototaxis. Appl. Phys. Lett. 2007, 90, 263901. [CrossRef]

31. Weibel, D.B.; Garstecki, P.; Ryan, D.; DiLuzio, W.R.; Mayer, M.; Seto, J.E.; Whitesides, G.M. Microoxen: Microorganisms to move microscale loads. Proc. Natl. Acad. Sci. USA 2005, 102, 11963-11967. [CrossRef] [PubMed]

32. Hatsuzawa, T.; Ito, D.; Nisisako, T.; Yanagida, Y. Micro-rotary ratchets driven by migratory phytoplankton with phototactic stimulus. Precis. Eng. 2017, 48, 107-113. [CrossRef]

33. Hatsuzawa, T.; Yamazaki, A.; Nisisako, T.; Yanagida, Y. Design and evaluation of artemia-driven micro ratchet gears. Sens. Actuators A 2015, 235, 182-186. [CrossRef]

34. Hatsuzawa, T.; Michishita, K.; Yanagida, Y. A reciprocating linear actuator driven by anti-phototaxis of plankton. Sens. Actuators A 2013, 201, 316-320. [CrossRef]

35. Stanton, M.M.; Park, B.-W.; Miguel-López, A.; Ma, X.; Sitti, M.; Sánchez, S. Biohybrid microtube swimmers driven by single captured bacteria. Small 2017, 13, 1603679. [CrossRef]

36. Stanton, M.M.; Simmchen, J.; Ma, X.; Miguel-López, A.; Sánchez, S. Biohybrid janus motors driven by Escherichia coli. Adv. Mater. Interfaces 2016, 3, 1500505. [CrossRef]

37. Di Leonardo, R.; Angelani, L.; Dell'Arciprete, D.; Ruocco, G.; Iebba, V.; Schippa, S.; Conte, M.P.; Mecarini, F.; De Angelis, F.; Di Fabrizio, E. Bacterial ratchet motors. Proc. Natl. Acad. Sci. USA 2010. [CrossRef]

38. Xu, H.; Medina-Sánchez, M.; Magdanz, V.; Schwarz, L.; Hebenstreit, F.; Schmidt, O.G. Sperm-hybrid micromotor for targeted drug delivery. ACS Nano 2018, 12, 327-337. [CrossRef]

39. Kirk, D.L. Seeking the ultimate and proximate causes of Volvox multicellularity and cellular differentiation. Integr. Comp. Biol. 2003, 43, 247-253. [CrossRef] [PubMed]

40. Drescher, K.; Leptos, K.C.; Tuval, I.; Ishikawa, T.; Pedley, T.J.; Goldstein, R.E. Dancing Volvox: Hydrodynamic bound states of swimming algae. Phys. Rev. Lett. 2009, 102, 168101. [CrossRef] [PubMed]

41. Solari, C.A.; Michod, R.E.; Goldstein, R.E. Volvox barberi, the fastest swimmer of the volvocales (chlorophyceae). J. Phycol. 2008, 44, 1395-1398. [CrossRef] [PubMed]

42. Eric, L.; Thomas, R.P. The hydrodynamics of swimming microorganisms. Rep. Prog. Phys. 2009, 72, 096601.

43. Brumley, D.R.; Polin, M.; Pedley, T.J.; Goldstein, R.E. Metachronal waves in the flagellar beating of Volvox and their hydrodynamic origin. J. R. Soc. Interface 2015, 12, 20141358. [CrossRef] [PubMed]

44. Drescher, K.; Goldstein, R.E.; Michel, N.; Polin, M.; Tuval, I. Direct measurement of the flow field around swimming microorganisms. Phys. Rev. Lett. 2010, 105, 168101. [CrossRef] [PubMed]

45. Drescher, K.; Goldstein, R.E.; Tuval, I. Fidelity of adaptive phototaxis. Proc. Natl. Acad. Sci. USA 2010, 107, 11171-11176. [CrossRef]

46. Charles, B.; Yohannes, D.; Kevin, K. Ultra-deep X-ray lithography of densely packed su-8 features: I. An su-8 casting procedure to obtain uniform solvent content with accompanying experimental results. J. Micromech. Microeng. 2005, 15, 1242.

47. Hernandez, C.J.; Mason, T.G. Colloidal alphabet soup: Monodisperse dispersions of shape-designed lithoparticles. J. Phys. Chem. C 2007, 111, 4477-4480. [CrossRef]

48. Nagai, M.; Oguri, M.; Shibata, T. Characterization of light-controlled Volvox as movable microvalve element assembled in multilayer microfluidic device. Jpn. J. Appl. Phys. 2015, 54, 067001. [CrossRef]

49. Dong, L.; Jiang, H. Autonomous microfluidics with stimuli-responsive hydrogels. Soft Matter 2007, 3, 1223-1230. [CrossRef]

(C) 2019 by the authors. Licensee MDPI, Basel, Switzerland. This article is an open access article distributed under the terms and conditions of the Creative Commons Attribution (CC BY) license (http://creativecommons.org/licenses/by/4.0/). 